\section{Impact of HER2 receptor status on axillary nodal burden in patients with non-luminal A invasive ductal breast carcinoma}

\author{
DOMAGOJ KUSTIC ${ }^{1}$, FRANJO LOVASIC ${ }^{2}$, \\ INGRID BELAC-LOVASIC ${ }^{3}$, MANUELA AVIROVIC ${ }^{4}$, \\ ALEN RUZIC ${ }^{5}$, SILVANA PETRETIC MAJNARIC ${ }^{1}$
}

\begin{abstract}
Background: Breast cancer $(B C)$ is the most common malignancy in women. Aim: To assess the impact of HER2 status on axillary lymph node (ALN) involvement in patients with invasive ductal carcinoma of no special type (IDCNST) both at diagnosis and during the 4-year postoperative period. Patients and Methods: We retrospectively included 375 women with an early clinical stage of non-luminal IDC-NST who between 2007 and 2013 underwent breast surgery at a clinical hospital. They were divided into phenotype-based groups: HR+HER2-, HR+HER2+, HR-HER2+ and HR-HER2-. Only patients with sentinel lymph node (SLN) macrometastases underwent ALN dissection. If $>3$ ALNs were positive, radiotherapy was delivered. All patients were treated with chemotherapy, HER2+BC patients received trastuzumab, and hormone receptor (HR)-positive BC patients received hormonal therapy. Results: Larger tumor size, higher grade, HR+, HER2+ status, and lymphovascular invasion (LVI) were predictive for ALN metastases at diagnosis. The poorest overall, disease-free, and distant recurrence-free survival (OS, DFS, DRFS) were found in the HR-HER2-group, while the poorest locoregional recurrence-free survival (LRFS) was observed in HR-HER2+ and HR-HER2-groups. HER2 status was not predictor of survival. Conclusions: HER2+ status was predictive for ALN involvement at diagnosis but had no effect on 4-year LRFS in these patients.
\end{abstract}

(Rev Med Chile 2019; 147: 557-567)

Key words: Breast Neoplasms; Receptor, ErbB-2; Sentinel Lymph Node; Trastuzumab.

\section{Receptores HER2, compromiso ganglionar axilar y sobrevida en mujeres con cáncer de mama ductal invasivo}

Antecedentes: El cáncer de mama es el tumor maligno más común en mujeres. Objetivo: Conocer el impacto del estado HER2 sobre el compromiso ganglionar axilar al momento del diagnóstico y durante los primeros cuatro años después de la cirugía en mujeres con carcinoma ductal invasivo de tipo no especial (IDC-NST). Pacientes y Métodos: Incluimos retrospectivamente a 375 mujeres en etapas clínicas iniciales de IDC-NST que fueron operadas en un
${ }^{1}$ Clinical Department of Nuclear Medicine, Clinical Hospital Center Rijeka, Rijeka, Croatia. ${ }^{2}$ Clinic for Surgery, Clinical Hospital Center Rijeka, Rijeka, Croatia.

${ }^{3}$ Clinic for Radiotherapy and Oncology, Clinical Hospital Center Rijeka, Rijeka, Croatia.

${ }^{4}$ Department of General

Pathology and Pathological Anatomy, Faculty of Medicine, University of Rijeka, Rijeka, Croatia.

${ }^{5}$ Department of Cardiology, Faculty of Medicine, University of Rijeka, Rijeka, Croatia.

Funding and disclosure: No sources of funding were used to assist in the work's inception, protocol design, data collection and the preparation of this manuscript. No conflicts of interest are relevant to the content of this manuscript.

Recibido el 19 de febrero de 2019, aceptado el 30 de abril de 2019.

\section{Correspodence to:}

Domagoj Kustic, MD, Clinical Department of Nuclear Medicine,

Clinical Hospital Center Rijeka, Krešimirova 42, 51000 Rijeka, Croatia.

domagoj.kustic.d@gmail.com 
hospital clínico. Ellas se dividieron en grupos de acuerdo al fenotipo: HR+HER2-, HR+HER2+, HR-HER2+y HR-HER2-. La disección de ganglios axilares se efectuó solo en las pacientes con macrometástasis en el ganglio centinela. Si había más de tres ganglios comprometidos, se efectuó radioterapia. Todas las pacientes se trataron con quimioterapia. Las pacientes HER2+ recibieron trastuzumab y las pacientes HR+ recibieron hormonoterapia. Resultados: Tumores más grandes, de mayor grado de malignidad, HR+, HER2+ y la invasión linfovascular fueron predictivos de la presencia de metástasis axilares al momento del diagnóstico. La sobrevida más baja se observó en pacientes HR-HER2+. La sobrevida libre de recurrencia locorregional más baja, se observó en pacientes HR-HER2+y HR-HER2-. HER2 no fue predictor de sobrevida. Conclusiones: En estas mujeres, HER2+ fue predictor de la presencia de compromiso ganglionar axilar al momento del diagnóstico pero no de la sobrevida a cuatro años.

Palabras clave: Ganglio Linfático Centinela; Neoplasias de la Mama; Trastuzumab; Receptor ErbB-2.

B reast cancer $(\mathrm{BC})$ is the most common malignancy in women worldwide ${ }^{1}$. Invasive ductal carcinoma of no special type (IDCNST) is the most common BC type ${ }^{2}$. According to the St. Gallen Conference 2015, BC molecular subtypes are defined by the status of estrogen and progesterone receptors (ER, PR), Ki-67 index, and human epidermal growth factor receptor 2 (HER2) status. As well as these parameters, a patient's age, the tumor size, the number of positive axillary lymph nodes (ALNs), and lymphovascular invasion (LVI) have an influence on the disease course, as well as on overall, disease-free, locoregional recurrence-free, and distant recurrence-free survival (OS, DFS, LRFS, DRFS) ${ }^{3}$.

HER2, that is overexpressed in about $20 \%$ of $\mathrm{BCs}$, is a predictor of early recurrence. These are commonly high-grade tumors, relatively resistant to hormonal therapy (HT). However, in HER2+ BC patients, HER2-targeted monoclonal antibody therapy, when added to adjuvant chemotherapy, yields improved survival benefits ${ }^{4}$.

In $\mathrm{BC}$, the disease staging and treatment are determined by ALN status. Sentinel lymph node (SLN) is the first lymph node (LN) in the drainage pathway of the area where a tumor is localized. SLN biopsy (SLNB) is the gold standard for axillary staging, with the purpose of avoiding unnecessary ALN dissections (ALNDs), along with associated comorbidities ${ }^{5}$. In the case of either negative SLNB or SLN involved with micrometastasis (0.2-2 $\mathrm{mm}$ in size) in early-stage BC patients, ALND is no longer indicated ${ }^{6}$. New studies showed that neither patients with 1 or 2 SLNs involved with macrometastases $(>2 \mathrm{~mm}$ in size) without extranodal extension (ENE) benefited from ALND if adjuvant radiotherapy (RT) was delivered ${ }^{7}$.

Tumor size, histologic grade, and LVI were recognized as independent predictors of ALN involvement ${ }^{8}$. A lower risk of early recurrence was observed in hormone receptor (HR)-positive and HER2-negative tumors, while a positive correlation was observed between $\mathrm{Ki}-67$ and the risk of early recurrence ${ }^{9,10}$. The impact of both HER2 status and molecular subtype on ALN involvement in early-stage BC, however, remains unclear, as inconclusive results have been obtained ${ }^{11-15}$.

The study objective was to assess the prognostic relevance of HER2 status in patients with an early clinical stage of IDC-NST in whom, following SLNB, ALND was performed only in the case of macrometastatic SLNs, with focuses on ALN involvement at diagnosis and on LRFS following postoperative systemic treatment. Comparisons, based on different HER2 status, were made between the subgroups homogenized according to HR status (positive/negative), Ki-67 $(<20 \% / \geq 20 \%)$, and to adjuvant treatment that the patients underwent following surgery (RT, HT, chemotherapy).

\section{Patients and Methods}

The research adhered to ethical policies and was approved by the Clinical Hospital Center 
Rijeka (CHCR) Ethics Committee. All subjects provided written informed consent. The inclusion criteria were: adult women with newly diagnosed IDC-NST in clinical stage T1-T2, with no clinical or ultrasonographic evidence of ALN involvement and no distant spread of the disease at diagnosis, who between 2007 and 2013 underwent breast surgery at the CHCR. We did not include: patients with locoregional BC recurrence, bilateral or multicentric BC, patients who received neoadjuvant RT, chemotherapy or HT, or those who had any other malignancies. Since luminal A, according to phenotypic features, treatment and prognosis, significantly differs from the remaining subtypes, patients with luminal A tumors were not included ${ }^{16}$.

A total of 185 HER2-positive IDC-NST patients met the inclusion criteria and were enrolled. For the purpose of forming approximately equally sized HER2+ and HER2- cohorts, 190 HER2-negative IDC-NST patients were also included. According to molecular subtype, the participants were split into groups: HR+/HER2-, HR+/ HER2+, HR-/HER2+, HR-/HER2-. Each group was divided into 2 subgroups according to Ki-67 $<20 \%$ or $\geq 20 \%$. The subgroups with matching HR status, matching Ki-67, and different HER2 statuses were eligible for comparison.

Following SLN scintigraphy with Tc-99m nanocolloid, the subjects underwent breast surgery and SLNB. SLNs were analyzed intraoperatively by imprint cytology (IC) and frozen section (FS) examinations. A positive IC, followed by positive FS, led to complete ALND during the first operation. The postoperative SLNB evaluation included hemalaun-eosin (HE) and pan-cytokeratin immunohistochemistry (IHC) ${ }^{17}$. If, after negative intraoperative IC and FS, SLN macrometastases had been confirmed by either HE or IHC, ALND was performed in a second operation. If SLNB was both positive for micrometastasis and ENE-negative, ALND was omitted ${ }^{6}$.

Based on the greatest dimension, the tumor size and the pathologic stage ( $\mathrm{pT}$ ) were determined $^{18}$. The histologic grade was determined according to the Elston and Ellis modified Bloom-Richardson system ${ }^{19}$. ER, PR and HER2 status, as well as Ki-67, were evaluated by $\mathrm{IHC}^{20,21}$. A tumor was considered HR-positive when either ER or PR status was positive (if at least 1\% of tumor cells stained ER- or PR-positive). A HR-negative tumor was defined as both ER- and
PR-negative. HER2-stained slides were evaluated by three expert pathologists at CHCR using light microscopy. In IHC-evaluated HER2-equivocal cases, HER2 status was confirmed by fluorescence in situ hybridization ${ }^{20,22}$.

Besides the above-mentioned parameters, information retrieved from the patients' records also included: age at diagnosis, type of surgery, the number of removed SLNs, the size of SLN metastatic deposit, the presence of LVI, and ENE in SLNs. In patients who underwent ALND, the ratio of macrometastatic LNs to the total LNs removed (lymph node ratio, LNR) was determined.

All subjects were to receive phenotype-based postoperative systemic treatment according to the St. Gallen Conference recommendations ${ }^{3,16}$. Patients with $\mathrm{HR}+$ tumors were treated with adjuvant HT based on menopausal status. All patients were treated with chemotherapy (with either 6 cycles of cyclophosphamide, 5-fluorouracil, and an anthracycline, or 4 cycles of cyclophosphamide and anthracycline followed by paclitaxel). Patients with HER2+ tumors were to receive 12-month trastuzumab treatment after receiving anthracycline chemotherapy ${ }^{16}$. If breast-conserving surgery was carried out, adjuvant RT to the whole breast was delivered. Patients who underwent mastectomy received no RT unless they had $>3$ positive $\mathrm{ALNs}^{16,17}$.

During the 4-year postoperative period, history taking and physical exams were conducted at 6-month intervals by oncologists at CHCR. Breast and axillary ultrasound examinations were being performed, along with cancer antigen 15-3 (CA 15-3) and carcinoembryonic antigen (CEA) being measured, on a 6-month basis. Mammography, chest radiography and abdominal ultrasound were performed on a 12-month basis. OS, DFS, LRFS, and DRFS, were calculated as the time in months from the date of surgery, respectively to the date of death from any cause, to the date of recurrence at any site, to the date of locoregional recurrence (LRR), and to the date of distant recurrence (DR).

The data were analyzed using Statistica 13.1. (StatSoft Inc., Tulsa, USA) and MedCalc 12.1.3. (MedCalc Software, Mariakerke, Belgium). The results are reported using descriptive statistics. The Chi-square test was used for categorical data. To assess differences in LVI, LNR, and ENE+ SLNs, both depending on HER2 status and among IDCNST groups, a Kruskal-Wallis test was applied, 
followed by Mann-Whitney $U$ post-hoc analysis. The relationship between patient/tumor characteristics and ALN involvement was tested by univariate logistic regression, from which significant factors were entered into multivariable logistic regression. Kaplan-Meier curves and the log-rank test were used to analyse differences in OS, DFS, LRFS, and DRFS among groups and subgroups. Based on Kaplan-Meier estimates, the proportions of subjects experiencing events at 4 years were calculated. Correlations between different variables were determined using Pearson's and Spearman's rank correlation coefficients. To determine which variables may predict OS, DFS, LRFS, and DRFS, multiple regression analysis was applied. The $\alpha$-error level was set to 0.05 .

\section{Results}

The total number of participants was 375 (median age 58 years; range 28-79). Patient and tumor characteristics are shown in Table 1.

As regards molecular subtypes, $126(33.6 \%)$, $123(32.8 \%), 62(16.5 \%)$, and $64(17.1 \%)$ were
HR+HER2-, HR+HER2+, HR-HER2+, and HRHER2-, respectively. A mastectomy was carried out in 97 patients $(25.87 \%)$, while 278 (74.13\%) underwent breast-conserving surgery. Sixty-seven patients (17.87\%) underwent postmastectomy RT due to $>3$ positive ALNs. The mean tumor size was $2.60 \mathrm{~cm}$ (range $0.4-6.9)$. The mean number of LNs removed was 2.11 (range 1-8) for SLN dissection and 18.80 (range 11-22) for ALND.

Within 4 years after surgery, 60 patients $(16.00 \%)$ died, while $66(17.60 \%)$ experienced recurrences, 16 of which $(24.24 \%)$ were locoregional ( 8 in the axilla; 8 in the breast), and 50 $(75.76 \%)$ distant.

When compared to the subjects without recurrences, in subjects with recurrences macrometastatic SLNs were more frequent $(68.18 \%$ vs $33.01 \%$, $P<0.001$ ), while micrometastatic SLNs were less frequent $(1.52 \%$ vs $12.62 \%, P=0.008)$, as well as negative SLNs $(30.30 \%$ vs $54.37 \%, P<0.001)$. SLN macrometastases were found in 147 patients (39.2\%), which led to ALND. Of the latter, 75 $(51.02 \%)$ had positive non-sentinel ALNs, 45 (30.61\%) experienced recurrences, in $8(5.44 \%)$ LRR occurred, and 44 (29.93\%) had ENE+ SLNs.

Table 1. Patient and tumor characteristics by IDC-NST molecular subtype

\begin{tabular}{|c|c|c|c|c|c|c|c|}
\hline & & $\mathbf{N}$ & HR+HER2- & HR + HER2 + & HR-HER2 + & HR-HER2- & $\mathbf{P}$ \\
\hline Age (years) & $\begin{array}{l}\leq 50 \\
>50\end{array}$ & $\begin{array}{l}134(35.7) \\
241(64.3)\end{array}$ & $\begin{array}{l}32(25.4) \\
94(74.6)\end{array}$ & $\begin{array}{l}40(32.5) \\
83(67.5)\end{array}$ & $\begin{array}{l}31(50.0) \\
31(50.0)\end{array}$ & $\begin{array}{l}31(48.4) \\
33(51.6)\end{array}$ & $<0.001$ \\
\hline pT stage & $\begin{array}{l}\text { pT1 } \\
\text { pT2 } \\
\text { pT3 }\end{array}$ & $\begin{array}{r}177(47.2) \\
139(37.1) \\
59(15.7)\end{array}$ & $\begin{array}{l}64(50.8) \\
46(36.5) \\
16(12.7)\end{array}$ & $\begin{array}{l}54(43.9) \\
45(36.6) \\
24(19.5)\end{array}$ & $\begin{array}{r}28(45.2) \\
25(40.3) \\
9(14.5)\end{array}$ & $\begin{array}{l}31(48.4) \\
23(35.9) \\
10(15.6)\end{array}$ & 0.829 \\
\hline pN stage & $\begin{array}{l}\text { pN0 } \\
\text { pN1mi } \\
\text { pN1 } \\
\text { pN2 } \\
\text { pN3 }\end{array}$ & $\begin{array}{r}188(50.1) \\
40(10.7) \\
80(21.3) \\
41(10.9) \\
26 \quad(6.9)\end{array}$ & $\begin{array}{l}33(26.2) \\
30(23.8) \\
33(26.2) \\
17(13.5) \\
13(10.3)\end{array}$ & $\begin{array}{r}48(39.0) \\
6(4.9) \\
39(31.7) \\
19(15.4) \\
11(8.9)\end{array}$ & $\begin{array}{rr}47 & (75.8) \\
2 & (3.2) \\
7 & (11.3) \\
4 & (6.5) \\
2 & (3.2)\end{array}$ & $\begin{aligned} 60 & (93.7) \\
2 & (3.1) \\
1 & (1.6) \\
1 & (1.6) \\
0 & (0)\end{aligned}$ & 0 \\
\hline Grade & $\begin{array}{l}\text { I } \\
\text { II } \\
\text { III }\end{array}$ & $\begin{array}{l}125(33.3) \\
132(35.2) \\
118(31.5)\end{array}$ & $\begin{array}{l}40(31.8) \\
45(35.7) \\
41(32.5)\end{array}$ & $\begin{array}{l}45(36.6) \\
49(39.8) \\
29(23.6)\end{array}$ & $\begin{array}{l}24(38.7) \\
15(24.2) \\
23(37.1)\end{array}$ & $\begin{array}{l}16(25.0) \\
23(35.9) \\
25(39.1)\end{array}$ & 0.146 \\
\hline $\mathrm{Ki}-67$ & $\begin{array}{l}<20 \% \\
\geq 20 \%\end{array}$ & $\begin{array}{l}110(29.3) \\
265(70.7)\end{array}$ & $\begin{array}{l}54(42.9) \\
72(57.1)\end{array}$ & $\begin{array}{l}51(41.5) \\
72(58.5)\end{array}$ & $\begin{array}{rr}1 & (1.6) \\
61 & (98.4)\end{array}$ & $\begin{array}{rr}4 & (6.2) \\
60 & (93.8)\end{array}$ & $<0.001$ \\
\hline LVI & $\begin{array}{l}\text { Negative } \\
\text { Positive }\end{array}$ & $\begin{array}{l}272(72.5) \\
103(27.5)\end{array}$ & $\begin{array}{l}93(73.8) \\
33(26.2)\end{array}$ & $\begin{array}{l}82(66.7) \\
41(33.3)\end{array}$ & $\begin{array}{l}41(66.1) \\
21(33.9)\end{array}$ & $\begin{array}{r}56(87.5) \\
8(12.5)\end{array}$ & 0.014 \\
\hline ENE & $\begin{array}{l}\text { Negative } \\
\text { Positive }\end{array}$ & $\begin{array}{r}331(88.3) \\
44(11.7)\end{array}$ & $\begin{array}{r}109(86.5) \\
17(13.5)\end{array}$ & $\begin{array}{r}103(83.7) \\
20(16.3)\end{array}$ & $\begin{array}{r}56(90.3) \\
6 \quad(9.7)\end{array}$ & $\begin{array}{r}63(98.4) \\
1 \quad(1.6)\end{array}$ & 0.024 \\
\hline
\end{tabular}

Note: IDC-NST, invasive ductal carcinoma of no special type; HR, hormone receptor; HER2, human epidermal growth factor receptor 2; LVI, lymphovascular invasion; ENE, extranodal extension. 
Forty subjects $(10.67 \%)$ had micrometastatic SLNs. Of these, one developed a recurrence, at a distant site. Out of 188 subjects with negative SLNs, 20 (10.64\%) developed recurrences (8 LRR, 12 DR).

In patients with ENE+ SLNs, both the proportion of $\mathrm{pN} 2-\mathrm{pN} 3$ stages and the proportion of cases with positive non-sentinel ALNs were higher compared to patients with macrometastatic, ENE-negative SLNs $(86.36 \%$ vs $35.92 \%, 84.09 \%$ vs $30.10 \%$; both $P<0.001$ ).

The incidence of LVI was lower in HER2-negative compared to HER2-positive IDC-NST, and LNR was lower in HR-HER2- compared to other subtypes (both $P<0.05$ ).

The univariate logistic analysis showed that patients with larger tumor size, higher grade, HR+ status, HER2+ status, and LVI had a higher risk of ALN metastases (Table 2). In the multivariable logistic analysis, after adjusting for tumor size, grade, HR, HER2 and LVI (model A), larger tumor size, higher grade, HR+ status, HER2+ status, and LVI were predictive for ALN metastases. After adjusting for tumor size, grade, LVI, and IDC-NST subtype (model B), larger tumor size, higher grade, and LVI remained predictive for ALN metastases (Table 3).

Kaplan-Meier analyses demonstrated differences in OS $(P=0.002)$, DFS $(P=0.002)$, LRFS $(P=0.045)$, and DRFS $(P=0.044)$ among IDCNST subtypes. The poorest OS, DFS, and DRFS were noted in HR-HER2- subtype. The poorest LRFS was seen in HR-HER2+ and HR-HER2subtypes (Table 4, Figure 1).

OS, DFS, LRFS, and DRFS were comparatively analysed between the subgroups with matching HR, matching Ki-67, and different HER 2 statuses (with the exception of the HR-HER $2+/<20$ and HR-HER2- $/<20$ pair, due to the small sample size), showing no significant differences (Table 5).

Table 2. Univariate analysis of clinicopathological factors associated with axillary lymph node metastases at diagnosis of IDC-NST

\begin{tabular}{|c|c|c|c|c|}
\hline & & OR & $95 \% \mathrm{Cl}$ & $\mathbf{P}$ \\
\hline Age (years) & $\begin{array}{l}\leq 50 \\
>50\end{array}$ & $\begin{array}{l}1 \\
1.132\end{array}$ & $0.733-1.748$ & 0.577 \\
\hline Tumor size & $\begin{array}{l}\text { pT1 } \\
\text { pT2 } \\
\text { pT3 }\end{array}$ & $\begin{array}{l}1 \\
3.168 \\
5.031\end{array}$ & $\begin{array}{l}1.960-5.121 \\
2.686-9.422\end{array}$ & $\begin{array}{l}<0.001 \\
<0.001\end{array}$ \\
\hline Grade & $\begin{array}{l}\text { I } \\
\text { II } \\
\text { III }\end{array}$ & $\begin{array}{l}1 \\
3.030 \\
5.474\end{array}$ & $\begin{array}{l}1.726-5.321 \\
3.079-9.732\end{array}$ & $\begin{array}{l}<0.001 \\
<0.001\end{array}$ \\
\hline$H R$ & $\begin{array}{l}\text { Negative } \\
\text { Positive }\end{array}$ & $\begin{array}{l}1 \\
8.349\end{array}$ & $4.610-15.119$ & $<0.001$ \\
\hline HER2 & $\begin{array}{l}\text { Negative } \\
\text { Positive }\end{array}$ & $\begin{array}{l}1 \\
1.531\end{array}$ & $1.009-2.323$ & 0.045 \\
\hline Ki-67 & $\begin{array}{l}<20 \\
\geq 20\end{array}$ & $\begin{array}{l}1 \\
0.657\end{array}$ & $0.419-1.031$ & 0.068 \\
\hline Lymphovascular invasion & $\begin{array}{l}\text { Negative } \\
\text { Positive }\end{array}$ & $\begin{array}{l}1 \\
6.101\end{array}$ & $3.708-10.038$ & $<0.001$ \\
\hline Subtype & $\begin{array}{l}\text { HR + HER2 - vs HR-HER2- } \\
\text { HR + HER2 + vs HR-HER2- } \\
\text { HR-HER2 + vs HR-HER2- } \\
\text { HR + HER2 - vs HR + HER2 + } \\
\text { HR + HER2 - vs HR-HER2 + } \\
\text { HR + HER2 + vs HR-HER2 + }\end{array}$ & $\begin{array}{r}31.000 \\
39.611 \\
8.225 \\
0.783 \\
3.769 \\
4.816\end{array}$ & $\begin{array}{l}7.266-132.258 \\
9.269-169.279 \\
1.772-38.178 \\
0.475-1.289 \\
1.864-7.622 \\
2.374-9.773\end{array}$ & $\begin{aligned}< & 0.001 \\
< & 0.001 \\
& 0.007 \\
& 0.335 \\
< & 0.001 \\
< & 0.001\end{aligned}$ \\
\hline
\end{tabular}

Note: IDC-NST, invasive ductal carcinoma of no special type; HR, hormone receptor; HER2, human epidermal growth factor receptor 2; OR, odds ratio; $\mathrm{Cl}$, confidence interval. 
Table 3. Multivariable analysis of clinicopathological factors associated with axillary lymph node metastases at diagnosis of IDC-NST

\begin{tabular}{|c|c|c|c|c|}
\hline & & OR & $95 \% \mathrm{Cl}$ & $\mathbf{P}$ \\
\hline \multicolumn{5}{|l|}{ MODEL A } \\
\hline \multirow[t]{2}{*}{ Tumor size } & pT2 vs pT1 & 1.998 & $1.086-3.677$ & 0.026 \\
\hline & pT3 vs pT1 & 2.637 & $1.097-6.342$ & 0.030 \\
\hline \multirow[t]{2}{*}{ Grade } & || vs I & 2.481 & $1.262-4.879$ & 0.008 \\
\hline & |ll vs I & 7.928 & $3.541-17.751$ & $<0.001$ \\
\hline$H R$ & Positive vs Negative & 23.108 & $10.144-52.640$ & $<0.001$ \\
\hline HER2 & Positive vs Negative & 1.832 & $1.053-3.187$ & 0.032 \\
\hline Lymphovascular invasion & Positive vs Negative & 4.592 & $2.405-8.767$ & $<0.001$ \\
\hline \multicolumn{5}{|l|}{ MODEL B } \\
\hline \multirow[t]{2}{*}{ Tumor size } & pT2 vs pT1 & 2.001 & $1.086-3.686$ & 0.026 \\
\hline & pT3 vs pT1 & 2.705 & $1.117-6.551$ & 0.027 \\
\hline \multirow[t]{2}{*}{ Grade } & || vs I & 2.447 & $1.251-4.787$ & 0.009 \\
\hline & |II vs I & 7.435 & $3.336-16.574$ & $<0.001$ \\
\hline Lymphovascular invasion & Positive vs Negative & 4.264 & $2.223-8.178$ & $<0.001$ \\
\hline \multirow[t]{6}{*}{ Tumor subtype } & HR+HER2- vs HR-HER2- & 59.047 & $12.589-276.956$ & $<0.001$ \\
\hline & HR + HER2 + vs HR-HER2- & 86.746 & $17.907-420.226$ & $<0.001$ \\
\hline & HR-HER2 + vs HR-HER2- & 6.207 & $1.218-31.619$ & 0.028 \\
\hline & $\mathrm{HR}+\mathrm{HER} 2-$ vs HR + HER2 + & 0.681 & $0.372-1.246$ & 0.213 \\
\hline & HR + HER2- vs HR-HER2 + & 9.514 & $3.787-23.900$ & $<0.001$ \\
\hline & $\mathrm{HR}+\mathrm{HER} 2+$ vs HR-HER2 + & 13.977 & $5.292-36.914$ & $<0.001$ \\
\hline
\end{tabular}

Note: IDC-NST, invasive ductal carcinoma of no special type, HR, hormone receptor; HER2, human epidermal growth factor receptor 2; OR, odds ratio; $\mathrm{Cl}$, confidence interval

Table 4. Kaplan-Meier overall, disease-free, locoregional recurrence-free, and distant recurrence-free survival estimates in patients with non-luminal A IDC-NST by molecular subtype

\begin{tabular}{|lccccccccc|}
\hline Subtype & OS (\%) & $\mathbf{9 5 \%} \mathbf{C l}$ & DFS (\%) & $\mathbf{9 5 \%} \mathbf{C l}$ & LRFS (\%) & $\mathbf{9 5 \%} \mathbf{C l}$ & DRFS (\%) & $\mathbf{9 5 \%} \mathbf{C I}$ & $\mathbf{N}$ \\
\hline HR+HER2- & 89.68 & $84.37-94.99$ & 88.10 & $82.44-93.75$ & 97.62 & $94.96-100.3$ & 90.48 & $85.35-95.60$ & 126 \\
HR+HER2+ & 86.99 & $81.05-92.94$ & 86.18 & $80.08-92.28$ & 97.56 & $94.84-100.3$ & 88.62 & $83.01-94.23$ & 123 \\
HR-HER2 + & 77.42 & $67.01-87.83$ & 75.81 & $65.15-86.47$ & 91.94 & $85.16-98.71$ & 83.87 & $74.72-93.03$ & 62 \\
HR-HER2- & 70.31 & $59.12-81.51$ & 68.75 & $57.39-80.11$ & 92.19 & $85.61-98.76$ & 76.56 & $66.18-86.94$ & 64 \\
\hline
\end{tabular}

Note: IDC-NST, invasive ductal carcinoma of no special type; HR, hormone receptor; HER2, human epidermal growth factor receptor 2; OS, overall survival; DFS, disease-free survival; LRFS, locoregional recurrence-free survival; DRFS, distant recurrence-free survival; $\mathrm{Cl}$, confidence interval.

The multiple regression analysis suggested that histologic grade, HR status, LNR, LVI, molecular subtype, and tumor size, may predict OS, DFS,
LRFS, and DRFS, while age $(\leq 50 />50$ years $)$, HER2 status, Ki-67 $(<20 \% / \geq 20 \%)$, and ENE in SLNs were not predictive (Table 6). 
A

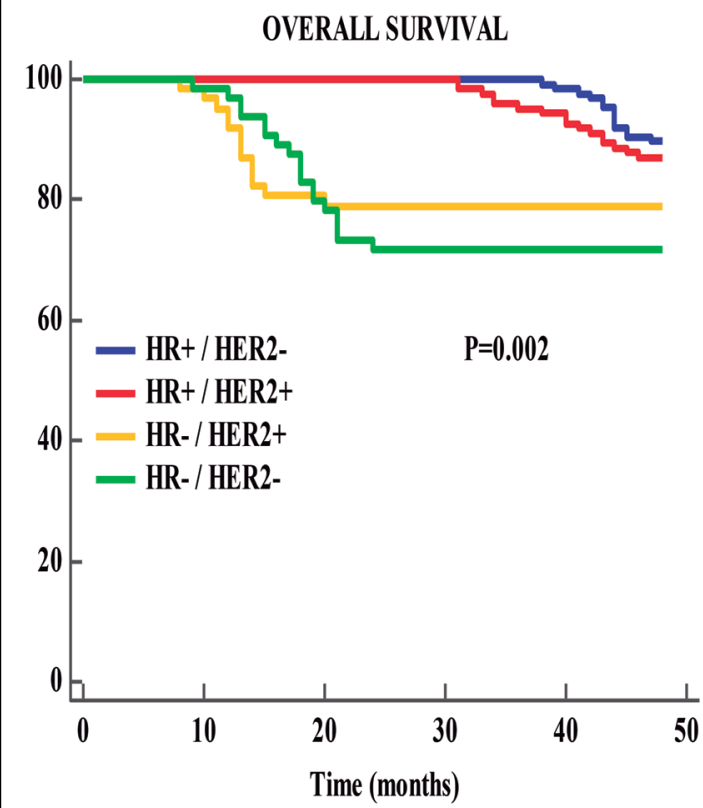

$\mathrm{C}$

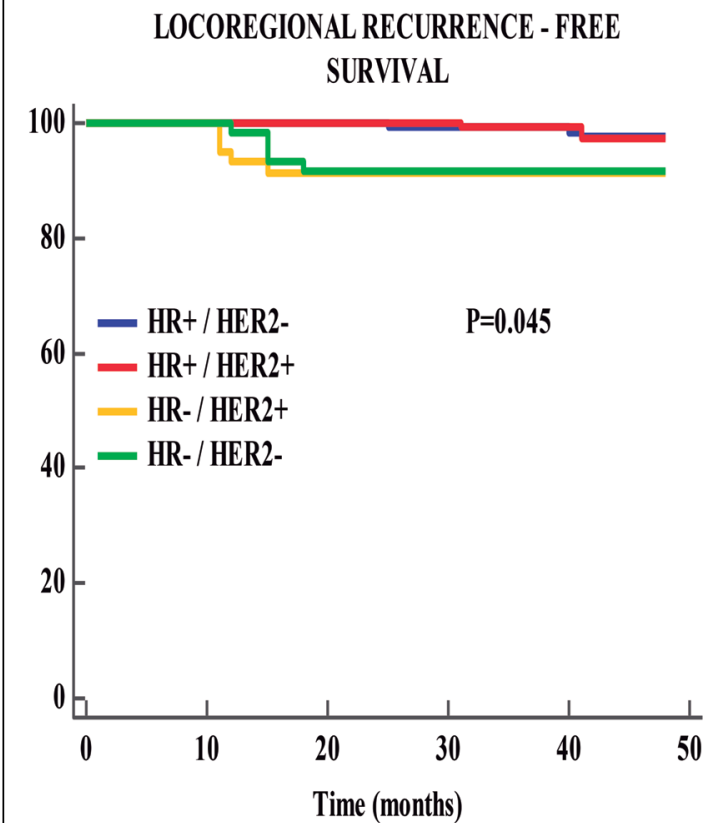

B

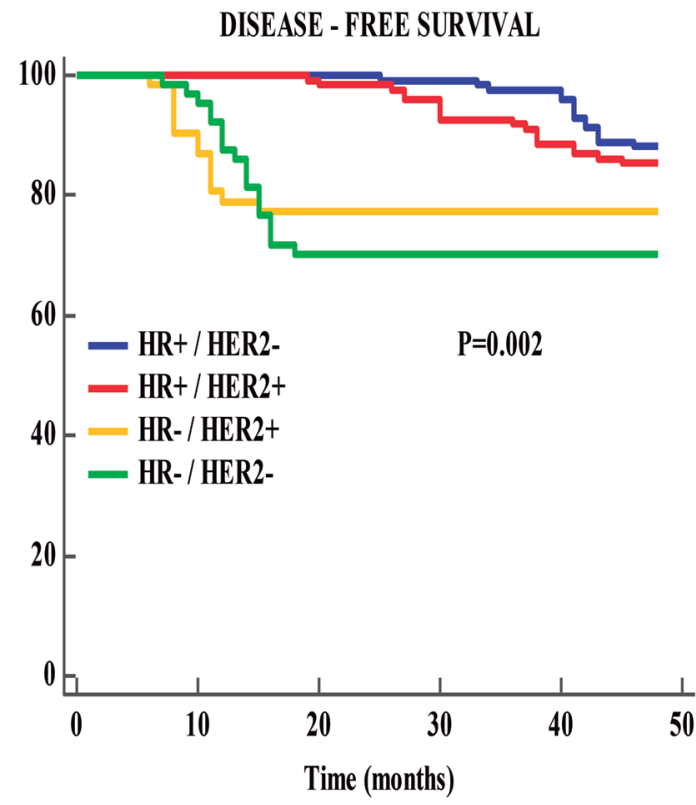

$\mathrm{D}$
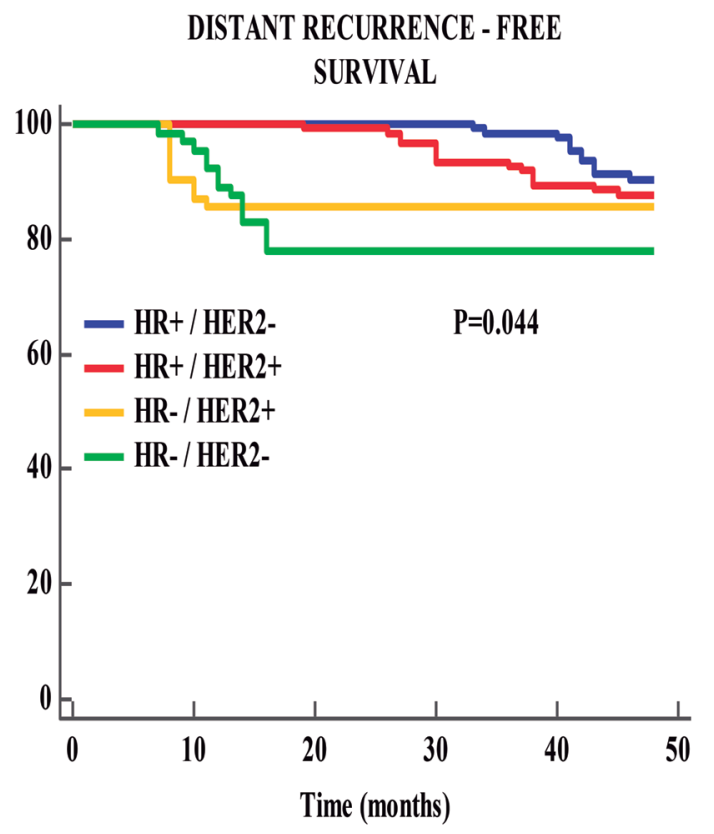

Figure 1. 
Table 5. Comparison of Kaplan-Meier overall, disease-free, locoregional recurrence-free, and distant recurrence-free survival estimates between IDC-NST patients with matching HR, matching Ki-67 and different HER2 statuses

\begin{tabular}{|c|c|c|c|c|c|c|c|c|c|}
\hline & OS $\left(0^{\circ}\right.$ & $95 \% \mathrm{Cl}$ & DFS (\%) & $95 \% \mathrm{Cl}$ & LRFS (\%) & $95 \% \mathrm{Cl}$ & DRFS (\%) & $95 \% \mathrm{Cl}$ & $\mathbf{N}$ \\
\hline$=R 2-1<20$ & 96.30 & $91.26-101.3$ & 96.30 & $91.26-101.3$ & 98.15 & $94.55-101.7$ & 98.15 & $94.55-101.7$ & 54 \\
\hline $\mathrm{HR}+\mathrm{HER} 2+/<20$ & 96.08 & $90.75-101.4$ & 92.16 & $84.78-99.54$ & 96.08 & $90.75-101.4$ & 96.08 & $90.75-101.4$ & 51 \\
\hline HR + HER2-/ $\geq 20$ & 83.33 & $74.73-91.94$ & 80.56 & $71.41-89.70$ & 95.83 & $91.22-100.5$ & 84.72 & 76.41-93.03 & 72 \\
\hline $\mathrm{HR}+\mathrm{HER} 2+1 \geq 20$ & 80.56 & $71.41-89.70$ & 79.17 & $69.79-88.55$ & 97.22 & $93.43-101.0$ & 81.94 & $73.06-90.83$ & 72 \\
\hline & 78.69 & 68.41-88.97 & 77.05 & $66.50-87.60$ & 91.80 & $84.92-98.69$ & 85.25 & $76.35-94.15$ & 61 \\
\hline HR-HER2- $/ \geq 20$ & 70.00 & $58.40-81.60$ & 66.67 & $54.74-78.60$ & 90.00 & $82.41-97.59$ & 76.67 & $65.96-87.37$ & 60 \\
\hline
\end{tabular}

Note: IDC-NST, invasive ductal carcinoma of no special type, HR, hormone receptor; HER2, human epidermal growth factor receptor 2; OS, overall survival; DFS, disease-free survival; LRFS, locoregional recurrence-free survival; DRFS, distant recurrence-free survival; $\mathrm{Cl}$, confidence interval.

Table 6. Significant clinicopathological predictors of overall, disease-free, locoregional recurrence-free, and distant recurrence-free survival in patients with non-luminal A IDC-NST evaluated by multiple regression analysis

\begin{tabular}{|lccccccc|}
\hline & Grade $(\boldsymbol{\beta})$ & HR $(\boldsymbol{\beta})$ & LNR $(\boldsymbol{\beta})$ & LVI $(\boldsymbol{\beta})$ & Subtype $(\boldsymbol{\beta})$ & Tumor size $(\boldsymbol{\beta})$ & R2/adjusted R2 \\
\hline OS & $-1.151^{*}$ & $4.961^{* *}$ & $-8.923^{* * *}$ & $-5.222^{* * *}$ & $-1.612^{*}$ & $-1.721^{* *}$ & $0.38 / 0.36^{* * *}$ \\
\hline DFS & $-1.308^{*}$ & $4.654^{*}$ & $-13.024^{* * *}$ & $-6.489^{* * *}$ & $-2.257^{*}$ & $-2.139^{* *}$ & $0.42 / 0.40^{* * *}$ \\
\hline LRFS & $-1.161^{*}$ & $5.655^{* *}$ & $-8.687^{* *}$ & $-6.019 * * *$ & $-1.592^{*}$ & $-1.938^{* *}$ & $0.39 / 0.37^{* * *}$ \\
\hline DRFS & $-1.298^{*}$ & $3.960^{*}$ & $-13.260^{* * *}$ & $-5.692^{* * *}$ & $-2.277^{*}$ & $-1.923^{* *}$ & $0.41 / 0.40^{* * *}$ \\
\hline
\end{tabular}

Note: IDC-NST, invasive ductal carcinoma of no special type; OS, overall survival; DFS, disease-free survival; LRFS, locoregional recurrence-free survival; DRFS, distant recurrence-free survival; HR, hormone receptors; LNR, lymph node ratio; LVI, lymphovascular invasion; $\beta$, standardized regression coefficient; $R^{2}$ /adjusted $R^{2}$, coefficient of determination / adjusted for the number of predictors in the model; the significance level: ${ }^{*} \mathrm{P}<0.05 ;{ }^{*} \mathrm{P}<0.01 ;{ }^{* * *} \mathrm{P}<0.001$

\section{Discussion and conclusions}

The molecular markers, ER, PR, HER2, and $\mathrm{Ki}-67$, as well as traditional factors, such as age, tumor size, histologic type, histologic grade, LN stage, and LVI, are widely used in the management and treatment of $\mathrm{BC}^{23,24}$. In HER2+ BC patients, HER2-targeted therapy increased 10-year OS and 10 -year DFS from $75.2 \%$ to $84.0 \%$, and from $62.2 \%$ to $73.7 \%$, respectively ${ }^{22,25}$. Since the results of the American College of Surgeons Oncology Group Z0011 trial were published, a less invasive approach in the management of the axilla has been adopted, while SLNB remained the gold standard for ALN staging ${ }^{5,7}$. Therefore, the impact of HER2 status on ALN involvement needs to be reevaluated.
According to our results, HER2+ status is predictive of ALN involvement at diagnosis of non-luminal A IDC-NST. Besides HER2+, HR+ status, molecular subtype, larger tumor size, higher grade, and LVI, were also associated with a higher risk of ALN metastases, while age ( $\leq 50 />50$ years) and Ki-67 $(<20 \% / \geq 20 \%)$ were not. Similar findings had been observed on larger samples ${ }^{8,24,26}$. Nevertheless, the relationship between HR status and ALN involvement is still under debate, as studies exist in which an inverse relationship between $\mathrm{HR}+$ status and ALN involvement was suggested ${ }^{27}$.

Inconsistent conclusions have been reported as to which BC subtype is the least associated with ALN metastases. Ahmed $\mathrm{AR}^{27}$ reported the highest likelihood of ALN metastasis in HR-HER2+, while Jones et al. ${ }^{11}$ found no association between the 
subtypes and ALN involvement. According to our results, in HR-HER2- subtype ALN involvement was the least frequent, LNR was significantly lower compared to other subtypes, while the incidence of LVI was lower compared to HER2+ subtypes. These results are consistent with those of Crabb et al. ${ }^{13}$ and $\mathrm{He} \mathrm{ZY}$ et al. ${ }^{24}$, suggesting that HR-HER2$\mathrm{BC}$ spreads through hematogenous rather than lymphatic routes.

As opposed to the majority of earlier research, patients with luminal A tumors were not included in this study. Despite that, the survival rates are comparable to those reported earlier, although the OS that we observed in HR-HER2+ and HRHER2- subtypes was somewhat lower ${ }^{28,29}$. This may be related to the smaller sample size and a relatively short postoperative observing period in our study. In agreement with former research, we found the poorest OS, DFS, and DRFS in the HRHER2- subtype, due to limited treatment options, while the poorest LRFS was noted in HR-HER2+ and HR-HER2- subtypes. Our results support those of Vuduc KD et al. ${ }^{30}$, where amongst those patients undergoing breast-conserving surgery, those with HR-HER2- and HR-HER2+ subtypes had a higher risk of LRR.

Of the markers defining BC subtype, only HR status had an influence on OS, DFS, LRFS, and DRFS. In contrast to the predictive capacity of HER2+ status for ALN involvement at diagnosis, no impact of HER2 status on the survival rates was observed. These results reflect the prognostic improvements as a result of HER2-targeted therapy. Although neither Ki-67 affected the survival rates, these results should be treated with caution, given that the majority of the subjects assigned to HRHER2+ and HR-HER2- groups had Ki-67 $\geq 20 \%$. Moreover, the prognostic role of Ki-67 has been suggested in previous papers ${ }^{8,9,21}$.

The tumor size is established as an independent predictor of survival in BC patients ${ }^{23}$. However, its prognostic role has been denied in some papers ${ }^{31}$. In this study, tumor size, as well as higher histologic grades, were negatively correlated with the survival rates ${ }^{8,13,14,18}$. Our results, showing that both the number of positive ALNs and the presence of LVI have a negative impact on the survival rates, are in line with previously published data ${ }^{32-36}$. In concordance with former observations, BC molecular subtype also affected survival ${ }^{12,24,31}$.

The data concerning the impact of age on the prognosis in $\mathrm{BC}$ patients are conflicting ${ }^{31,37}$. We observed no impact of age $(\leq 50 />50$ years $)$ on the survival rates. Note, however, that among our patients the number of those aged $\leq 50$ years was lower.

Although a negative impact of ENE in SLNs on survival has been suggested ${ }^{34}$, we observed none. Nevertheless, in our patients with ENE-positive SLNs, higher nodal stages ( $\mathrm{pN} 2-\mathrm{pN} 3$ ) were more frequent and non-sentinel ALNs were more often positive compared to the patients with macrometastatic, ENE-negative SLNs, indicating that ENE in SLNs affects survival indirectly, which is in accordance with the findings of Schwentner L et al. ${ }^{38}$.

In conclusion, in non-luminal A IDC-NST, an increased risk of ALN metastases at diagnosis is associated with HER2+ status. However, no impact of HER2 status on LRFS was observed. Besides the benefits of HT, chemotherapy and HER2-targeted therapy in HR+ and HER2+ BC patients, limited improvements have been achieved in HR-HER2+ subtype, in which LRFS is not better than in the HR-HER2- subtype. Therefore, in patients with HR-HER2+ subtype, better locoregional control is necessary.

Acknowledgments: We thank Sarah Caroline Czerny for the excellent technical assistance.

\section{References}

1. Bray F, Ferlay J, Soerjomataram I, Siegel RL, Torre LA, Jemal A. Global Cancer Statistics 2018: GLOBOCAN Estimates of Incidence and Mortality Worldwide for 36 Cancers in 185 Countries. CA Cancer J Clin 2018; 68 (6): 394-424.

2. Makki J. Diversity of breast carcinoma: Histological subtypes and clinical relevance. Clin Med Insights Pathol 2015; 8: 23-31.

3. Coates AS, Winer EP, Goldhirsch A, Gelber RD, Gnant $\mathrm{M}$, Piccart-Gebhart MJ, et al. Tailoring therapies-improving the management of early breast cancer: St Gallen International Expert Consensus on the Primary Therapy of Early Breast Cancer 2015. Ann Oncol 2015; 26 (8): 1533-46.

4. Gutiérrez C, Schiff R. HER2: Biology, detection, and clinical implications. Arch Pathol Lab Med 2011; 135 (1): 55-62.

5. Giuliano AE, McCall L, Beitsch P, Whitworth PW, Blumencranz P, Leitch AM, et al. Locoregional recurrence after sentinel lymph node dissection with or without 
axillary dissection in patients with sentinel lymph node metastases: The American College of Surgeons Oncology Group Z0011 randomized trial. Ann Surg 2010; 252 (3): 426-33.

6. Fournier K, Schiller A, Perry RR, Laronga C, Beenken SW, McGrath PC, et al. Micrometastasis in the sentinel lymph node of breast cancer does not mandate completion axillary dissection. Ann Surg 2004; 239 (6): 859-65.

7. Giuliano AE, Hunt KK, Ballman KV, Beitsch PD, Whitworth PW, Blumencranz PW, et al. Axillary dissection vs no axillary dissection in women with invasive breast cancer and sentinel node metastasis: A randomized clinical trial. JAMA 2011; 305 (6): 569-75.

8. Bader AA, Tio J, Petru E, Bühner M, Pfahlberg A, Volkholz $\mathrm{H}$, et al. T1 breast cancer: Identification of patients at low risk of axillary lymph node metastases. Breast Cancer Res Treat 2002; 76 (1): 11-7.

9. Joensuu K, Leidenius M, Kero M, Andersson LC, Horwitz KB, Heikkilä P. ER, PR, HER2, Ki-67 and CK5 in early and late relapsing breast cancer-reduced CK5 expression in metastases. Breast Cancer (Auckl) 2013; 7: 23-34.

10. Buonomo OC, Caredda E, Portarena I, Vanni G, Orlandi A, Bagni C, et al. New insights into the metastatic behavior after breast cancer surgery, according to well-established clinicopathological variables and molecular subtypes. PLoS One 2017; 12 (9): e0184680.

11. Jones T, Neboori H, Wu H, Yang Q, Haffty BG, Evans $\mathrm{S}$, et al. Are breast cancer subtypes prognostic for nodal involvement and associated with clinicopathologic features at presentation in early-stage breast cancer? Ann Surg Oncol 2013; 20 (9): 2866-72.

12. Reyal F, Rouzier R, Depont-Hazelzet B, Bollet MA, Pierga JY, Alran S, et al. The molecular subtype classification is a determinant of sentinel node positivity in early breast carcinoma. PLoS One 2011; 6 (5): e20297.

13. Crabb SJ, Cheang MC, Leung S, Immonen T, Nielsen TO, Huntsman DD, et al. Basal breast cancer molecular subtype predicts for lower incidence of axillary lymph node metastases in primary breast cancer. Clin Breast Cancer 2008; 8 (3): 249-56.

14. Van Calster B, Vanden Bempt I, Drijkoningen M, Pochet N, Cheng J, Van Huffel S, et al. Axillary lymph node status of operable breast cancers by combined steroid receptor and HER-2 status: Triple positive tumours are more likely lymph node positive. Breast Cancer Res Treat 2009; 113 (1): 181-7.

15. Si C, Jin Y, Wang H, Zou Q. Association between molecular subtypes and lymph node status in invasive breast cancer. Int J Clin Exp Pathol 2014; 7 (10): 6800-6.

16. Goldhirsch A, Wood WC, Gelber RD, Coates AS, Thür- limann B, Senn HJ. Progress and promise: Highlights of the international expert consensus on the primary therapy of early breast cancer 2007. Ann Oncol 2007; 18 (7): 1133-44.

17. Jonjic N, Mustac E, Bekafigo IS, Molek KR, Lovasic F, Lukanovic M, et al. [Analysis of sentinel lymph node in early breast cancer patient-Clinical Hospital Center Rijeka]. Lijec Vjesn 2013; 135 (7-8): 201-5.

18. Rakha EA, El-Sayed ME, Lee AHS, Elston CW, Grainge MJ, Hodi Z, et al. Prognostic significance of Nottingham histologic grade in invasive breast carcinoma. J Clin Oncol 2008; 26 (19): 3153-8.

19. Elston CW, Ellis IO. Pathological prognostic factors in breast cancer. I. The value of histological grade in breast cancer: experience from a large study with long-term follow-up. Histopathology 1991; 19 (5): 403-10.

20. Allred DC. Issues and updates: Evaluating estrogen receptor- $\alpha$, progesterone receptor, and HER2 in breast cancer. Mod Pathol 2010; 23 Suppl 2: S52-9.

21. De Azambuja E, Cardoso F, De Castro G, Colozza M, Mano MS, Durbecq V, et al. Ki-67 as prognostic marker in early breast cancer: A meta-analysis of published studies involving 12155 patients. Br J Cancer 2007; 96 (10): 1504-13.

22. Slamon DJ, Leyland-Jones B, Shak S, Fuchs H, Paton V, Bajamonde A, et al. Use of Chemotherapy plus a Monoclonal Antibody against HER2 for Metastatic Breast Cancer That Overexpresses HER2. N Engl J Med 2001; 344 (11): 783-92.

23. Elston CW, Ellis IO, Pinder SE. Pathological prognostic factors in breast cancer. Crit Rev Oncol Hematol 1999; 31 (3): 209-23.

24. He ZY, Wu SG, Yang Q, Sun JY, Li FY, Lin Q, et al. Breast Cancer Subtype is Associated With Axillary Lymph Node Metastasis: A Retrospective Cohort Study. Medicine (Baltimore) 2015; 94 (48): e2213.

25. Pérez EA, Romond EH, Suman VJ, Jeong JH, Sledge G, Geyer CE, et al. Trastuzumab plus adjuvant chemotherapy for human epidermal growth factor receptor 2 - Positive breast cancer: Planned joint analysis of overall survival from NSABP B-31 and NCCTG N9831. J Clin Oncol 2014; 32 (33): 3744-52.

26. Zhu X, Ying J, Wang F, Wang J, Yang H. Estrogen receptor, progesterone receptor, and human epidermal growth factor receptor 2 status in invasive breast cancer: a 3,198 cases study at National Cancer Center, China. Breast Cancer Res Treat 2014; 147 (3): 551-5.

27. Ahmed ARH. HER2 expression is a strong independent predictor of nodal metastasis in breast cancer. J Egypt Natl Canc Inst 2016; 28 (4): 219-27.

28. Hennigs A, Riedel F, Gondos A, Sinn P, Schirmacher 
P, Marmé F, et al. Prognosis of breast cancer molecular subtypes in routine clinical care: A large prospective cohort study. BMC Cancer 2016; 16 (1): 734.

29. Brouckaert O, Laenen A, Vanderhaegen J, Wildiers H, Leunen K, Amant F, et al. Applying the 2011 St Gallen panel of prognostic markers on a large single hospital cohort of consecutively treated primary operable breast cancers. Ann Oncol 2012; 23 (10): 2578-84.

30. Voduc KD, Cheang MCU, Tyldesley S, Gelmon K, Nielsen TO, Kennecke H. Breast cancer subtypes and the risk of local and regional relapse. J Clin Oncol 2010; 28 (10): 1684-91.

31. Kasangian AA, Gherardi G, Biagioli E, Torri V, Moretti A, Bernardin E, et al. The prognostic role of tumor size in early breast cancer in the era of molecular biology. PLoS One 2017; 12 (12): e0189127.

32. Woodward WA, Vinh-Hung V, Ueno NT, Cheng YC, Royce M, Tai P, et al. Prognostic value of nodal ratios in node-positive breast cancer. J Clin Oncol 2006; 24 (18): 2910-6.

33. Elkhodary TR, Ebrahim MA, Hatata EE, Niazy NA. Prognostic value of lymph node ratio in node-positive breast cancer in Egyptian patients. J Egypt Natl Canc Inst 2014; 26 (1): 31-5.
34. Aziz S, Wik E, Knutsvik G, Klingen TA, Chen Y, Davidsen $B$, et al. Extra-nodal extension is a significant prognostic factor in lymph node positive breast cancer. PLoS One 2017; 12 (2): e0171853.

35. Truong PT, Berthelet E, Lee J, Kader HA, Olivotto IA. The prognostic significance of the percentage of positive/dissected axillary lymph nodes in breast cancer recurrence and survival in patients with one to three positive axillary lymph nodes. Cancer 2005; 103 (10): 2006-14.

36. Rakha EA, Martin S, Lee AHS, Morgan D, Pharoah PDP, Hodi $Z$, et al. The prognostic significance of lymphovascular invasion in invasive breast carcinoma. Cancer 2012; 118 (15): 3678-80.

37. Chen HL, Zhou MQ, Tian W, Meng KX, He HF. Effect of age on breast cancer patient prognoses: A population-based study using the SEER 18 database. PLoS One 2016; 11 (10): e0165409.

38. Schwentner L, Dayan D, Wöckel A, Janni W, Kreienberg $\mathrm{R}$, Blettner $\mathrm{M}$, et al. Is extracapsular nodal extension in sentinel nodes a predictor for nonsentinel metastasis and is there an impact on survival parameters? A retrospective single center cohort study with 324 patients. Breast J 2018; 24 (4): 480-6. 